\title{
Super-Accurate Angular Encoder System with Multi-Detecting Heads Using VEDA Method
}

\author{
Nobuyuki ISHII**, Kayoko TANIGUCHI ${ }^{* * *}$, Kazuo YAMAZAKI ${ }^{\dagger}$ and Hideki AOYAMA**
}

\begin{abstract}
An angular encoder that can detect angular motion with ultrahigh accuracy is needed to enhance the precision for the angular motion axis of computer numerical control $(\mathrm{CNC})$ machine tools. Although a self-calibration method based on mounting multiple detecting heads has been proposed, it is impractical because the number of detecting heads needed to calibrate higher-order terms of the error components corresponds to the order. This paper presents a new self-calibration method for an angular encoder that uses fewer detecting heads. The method was compared with existing methods via simulation to confirm its feasibility. A prototype angle encoder was produced with six detecting heads to calibrate error component terms up to the 30th order. The error curves of the six sensor heads of the prototype encoder system were used to achieve a motion detection accuracy of $\pm 0.07 \mathrm{~s}$.
\end{abstract}

Key words: encoder, ultraprecision, compensation, angular measurement, rotary encoder

\section{Introduction}

\subsection{Needs and Trend}

The importance of precise angular measurement has increased for machine tools and precision measurement equipment. In particular, a rotary encoder needs to be developed to ensure high accuracy for machines.

Machine tools require further improvement regarding the processing accuracy of large round workpieces. For example, in the energy industry, the machine accuracy of components is assumed to directly affect energy the conversion efficiency and lifetime of components used in high-speed turbines or large gears of wind power generators, as shown in Fig. 1. 1),

${ }^{2)}$. There is strong demand for rotary encoders with better accuracy and resolution to serve as a measurement reference, especially for measurements of the surface accuracy of optical components or wafers that incorporate auto-collimators. These machine tools or precision measurement equipment use an auto-collimator by placing a polyhedron mirror over the machine. This method can measure a number of points identical to the number of mirrors. This is not enough to meet further demands for increased accuracy in the future.

A rotary encoder can achieve higher accuracy and resolution with high machining repeatability even if it is installed roughly without precise alignment. In response to the above requirements, multiple heads have been applied to a single rotary disc of an existing encoder to compensate for the calibration error. However, this increases the size of the encoder because of the

- Received on June 19, 2017

Accepted on May 17, 2018

- Full member, School of Integrated Design Engineering, Keio University (Hiyoshi Kohoku-ku, Yokohama,

Kanagawa 223-8522, Japan)

-. Magnescale Co., Ltd. (Suzukawa, Isehara-shi, Kanagawa 259-1146, Japan)

† Full member, Department of Mechanical Engineering, University of California at Berkeley, (Berkeley, CA 94720, USA) many heads required. Thus, this approach is impractical for implementation in a machine tool system.

\subsection{Conventional Technologies and Difficulties}

A rotary encoder has two error factors: the inherent error factor originates from the manufacturing process, whereas the acquired error factor originates from the process of installing the encoder into the user's machine ${ }^{3)}$. In order to eliminate the effect of these errors, several methods for self-calibrating a rotary encoder are available. Table 1 presents the major self-calibration methods for a single rotary encoder. For multiple reading head methods ${ }^{4}$, one head is installed as reference, and several heads are installed at intervals from the reference. Then, the calibration curve is calculated by obtaining Fourier components of the same order or less. The characteristics of the reference head affect the measurement because only two heads located at an interval of $180^{\circ}$ are used for measurement. Self $\mathrm{A}^{5)}$ is an enhanced equal division average (EDA) method ${ }^{7)}$ that uses two sets of rotary encoder for self-calibration. For this method, the number of heads should be one greater than the order of calibration. For better calibration accuracy, the number of heads should be increased. Combined SelfA ${ }^{6}$ enables higher-order calibration by

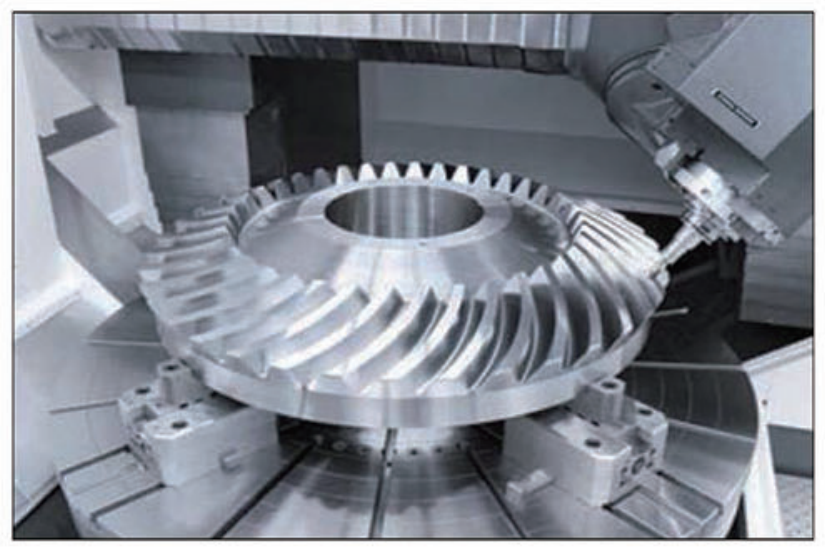

Fig. 1 Gear milling by five-axis machining center Source: DMG MORI Journal 
Table 1 Major methods of self-calibration for a single rotary encoder

\begin{tabular}{|c|c|c|c|}
\hline Method & $\begin{array}{l}\text { Divider } \\
\text { Method }\end{array}$ & $\begin{array}{l}\text { Multi-reading } \\
\text {-Method }\end{array}$ & $\begin{array}{c}\text { Equal } \\
\text { Deviation } \\
\text { Average } \\
\text { (EDA) } \\
\text { Method }\end{array}$ \\
\hline Feature & $\begin{array}{l}\text { Sequential } \\
\text { detection }\end{array}$ & \multicolumn{2}{|c|}{ Fourier component detection } \\
\hline Advantages & Short time & \multicolumn{2}{|c|}{$\begin{array}{c}\text { High accuracy } \\
\text { Short time } \\
\text { Self-diagnosis of the state }\end{array}$} \\
\hline Disadvantages & $\begin{array}{l}\text { Cumulative } \\
\text { uncertainty }\end{array}$ & $\begin{array}{c}\text { Effects of } \\
\text { characteristics of } \\
\text { the head }\end{array}$ & $\begin{array}{l}\text { Increase in } \\
\text { the number } \\
\text { of heads }\end{array}$ \\
\hline
\end{tabular}

combining multiple heads set at even intervals. The number of heads decreases with respect to the order of calibration.

\subsection{Purpose of this study}

In order to solve these problems, the authors devised a new method that the two neighboring sensors on a scale can generate calibration values that are the same as the case when the sensor heads are at equal intervals. The proposed method is called the virtual equal deviation average (VEDA) method. The purpose of this study is to confirm whether this proposed VEDA method can be calibrated to the order assumed under an ideal environment and to verify to what extent accuracy can be achieved with an actual sensor head. Specifically, the purpose is to achieve equal or better performance for each sensor head variation and installation error when calibrating by using the VEDA method the same order as SelfA method which based on EDA method similarly with a small number. And it is to clarify that accuracy of \pm 0.1 arcsec can be achieved with a sensor head actually used for prototyping.

\section{Principle of the VEDA Method}

To overcome these difficulties, a new method for self-calibration is proposed in this paper. In general, a sensor head at position $\theta$ with respect to the reference position of the detected value $H(\theta)$ of the rotary encoder can be represented as

$$
H(\theta)=\theta+\varepsilon(\theta)
$$

where $\theta$ is the angular position ( 0 to $2 \pi$ ) and $\varepsilon(\theta)$ is the periodic angle error term of the rotary encoder. The error term of the actual sensor has abroad frequency components. The error term is represented as

$$
\varepsilon(\theta)=\sum_{n=1}^{\infty} C_{n} \sin \left(n \theta+\alpha_{n}\right)
$$

where $n$ is a positive integer, $C_{n}$ is the amplitude of the $n$-th Fourier component, and $\alpha_{\mathrm{n}}$ is the phase of the $n$-th Fourier component. If the reference position is at the 0 th position of sensor heads positioned with $N$ units of equal intervals over one cycle, the detected value $H^{(N, k)}(\theta)$ of the $k$-th head can be represented as follows:

$$
\begin{aligned}
& H^{(N, k)}(\theta)=H\left(\theta+\frac{2 \pi k}{N}\right)-\frac{2 \pi k}{N}=\theta+\varepsilon\left(\theta+\frac{2 \pi k}{N}\right) \\
& (\mathrm{k}=0,1, \cdots, N-1)
\end{aligned}
$$

Consider two sensor heads separated by an angle of $2 \pi / N$ rad. The difference between the detected values $\delta^{((N, k))}(\theta)$ of the $k$ th and $(k-1)$ th sensor heads can be represented as follows:

$$
\begin{aligned}
\delta^{(N, k)}(\theta) & =H^{(N, k)}(\theta)-H^{(N, k-1)}(\theta) \\
& =\varepsilon\left(\theta+\frac{2 \pi k}{N}\right)-\varepsilon\left(\theta+\frac{2 \pi(k-1)}{N}\right) \\
& =\varepsilon\left(\left(\theta+\frac{2 \pi}{N}\right)+\frac{2 \pi(k-1)}{N}\right) \\
& -\varepsilon\left(\left(\theta+\frac{2 \pi}{N}\right)+\frac{2 \pi(k-2)}{N}\right) \\
& =\delta^{(N, k-1)}\left(\theta+\frac{2 \pi}{N}\right)
\end{aligned}
$$

Accordingly,

$$
\begin{aligned}
\delta^{(N, k)}(\theta) & =\delta^{(N, k-1)}\left(\theta+\frac{2 \pi}{N}\right) \\
& =\delta^{(N, k-2)}\left(\theta+2 \frac{2 \pi}{N}\right) \\
& =\delta^{(N, 1)}\left(\theta+\frac{2 \pi(k-1)}{N}\right)
\end{aligned}
$$

Therefore,

$$
H^{(N, k)}(\theta)=\delta^{(N, 1)}\left(\theta+\frac{2 \pi(k-1)}{N}\right)+H^{(N, k-1)}(\theta)
$$

where $\delta^{(N, 1)}\left(\theta+\frac{2 \pi(k-1)}{N}\right)$ is the phase shift of $\delta^{(N, 1)}(\theta)$.

Therefore, the error of the sensor head at $2 \pi k / N$ is described by the difference of error curve between 1st and 0th sensor head $\delta^{(N, 1)}(\theta) \varepsilon\left(\theta+\frac{2 \pi}{N}\right)$ and error curve between 1st sensor head:

$$
H^{(N, k)}(\theta)=\sum_{j=1}^{k-1} \delta^{(N, 1)}\left(\theta+\frac{2 \pi j}{N}\right)+H^{(N, 1)}(\theta)
$$

$$
(k=2,3, \ldots, N-1)
$$

In this manner, it is possible to calculate the detected value of the $N$ sensor heads arranged at equal intervals. The $N$ th-order components of the error $\varepsilon_{N}(\theta)$ can be described by using the principle of EDA method that "For any positive integer $N$, any arbitrary periodic curve generates a total $n$ of curves whose angle phase shifts by $2 \pi / N$ from the original curve. The averaged curve, from the total of $N$ curves, has components that are integral multiples of the $\mathrm{N}$-order Fourier components of the original curve." Therefore, the average of the detected values of the $N$ equally distributed sensor heads, $\bar{H}^{N}(\theta)$ is a detection value having an error of only the Nth order.

$$
\begin{aligned}
\bar{H}^{N}(\theta) & =\frac{1}{N} \sum_{k=0}^{N-1} H^{(N, k)}(\theta) \\
& =\theta+\sum_{n=1}^{\infty} \varepsilon\left(\theta+\frac{2 \pi k}{N}\right)
\end{aligned}
$$




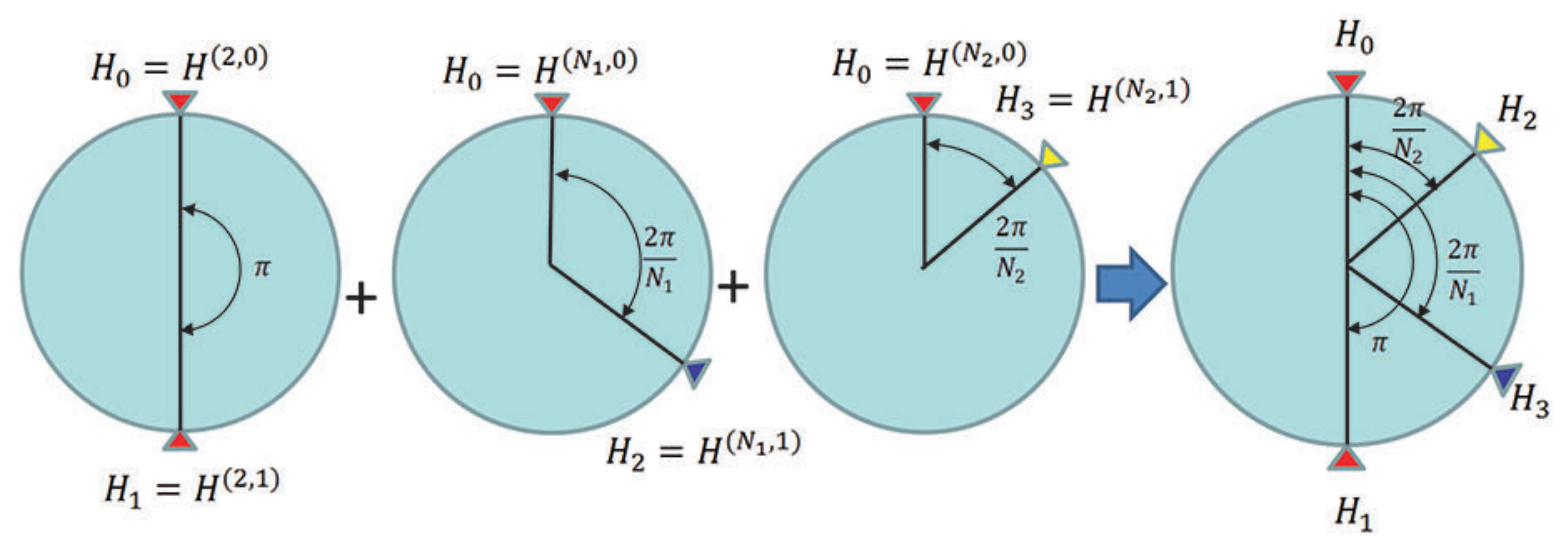

Fig. 2 Arrangement of sensors required for calibration companioning three pairs with VEDA method

$$
\begin{aligned}
\bar{H}^{N}(\theta) & =\frac{1}{N}\left\{H^{(N, 0)}(\theta)+(N-1) H^{(N, 1)}(\theta)\right. \\
& \left.+\sum_{k=2}^{N-1}\left\{\sum_{j=1}^{k-1} \delta^{(N, 1)}\left(\theta+\frac{2 \pi j}{N}\right)+\varepsilon\left(\theta+\frac{2 \pi}{N}\right)\right\}\right\} \\
=\frac{1}{N}\left\{H^{(N, 0)}(\theta)+(N-1) H^{(N, 1)}(\theta)\right. & \left.+\sum_{j=1}^{N-2}(N-1-j) \delta^{(N, 1, j)}(\theta)\right\}
\end{aligned}
$$

As in the case of calculating equation (7) using equation (6), the error $\varepsilon\left(\theta+\frac{2 \pi k}{N}\right)$ from the sensor head positioned at $2 \pi k / N$ can be also represented as $\delta^{(N, 1)}(\theta), \varepsilon(\theta)$ :

$$
\begin{gathered}
H^{(N, k)}(\theta)=\sum_{j=0}^{N-k-1} \delta^{(N, 1)}\left(\theta+\frac{2 \pi(k+j)}{N}\right)+H^{(N, 0)}(\theta) \\
(k=2, \ldots N-1)
\end{gathered}
$$

Substituting Eq. (11) into Eq. (9) yields

$$
\begin{aligned}
\bar{H}^{N}(\theta)=\frac{1}{N}\left\{(N-1) \varepsilon(\theta)+\varepsilon\left(\theta+\frac{2 \pi}{N}\right)\right. \\
\\
\left.\quad-\sum_{j=2}^{N-1}(j-1) \delta^{(N, 1)}\left(\theta+\frac{2 \pi}{N} j\right)\right\}
\end{aligned}
$$

Equations (9) and (11) represent the same thing, so the average can be described as follows:

$$
\begin{aligned}
\bar{H}^{N}(\theta) & =\frac{1}{2}\left\{H^{0}(\theta)+H^{1}(\theta)\right\} \\
& +\frac{1}{2 N}\left\{\sum_{j=1}^{N-1}(N-2 j) \delta^{(N, 1)}\left(\theta+\frac{2 \pi}{N} j\right)\right\}
\end{aligned}
$$

According to Eq. (12), the two neighboring sensors on a scale can generate calibration values that are the same as the case when the sensor heads are at equal intervals. This method allows for higher-order calibration with fewer sensor heads.

\section{Implementation of the VEDA Method}

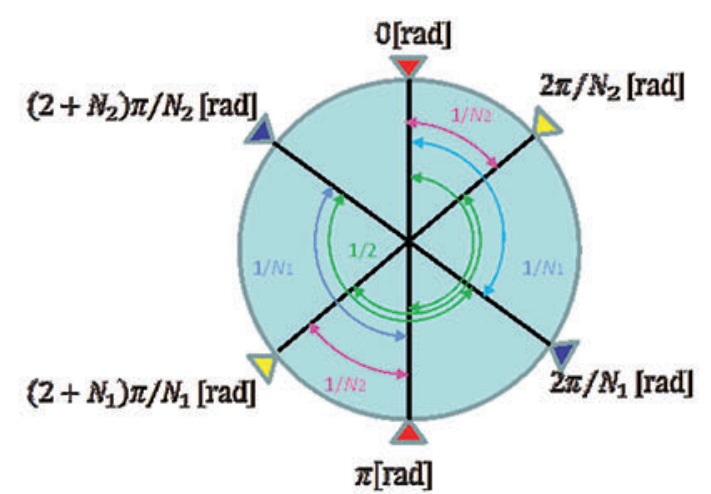

Fig. 3 Location of three pairs (six units) of sensor heads facing each other

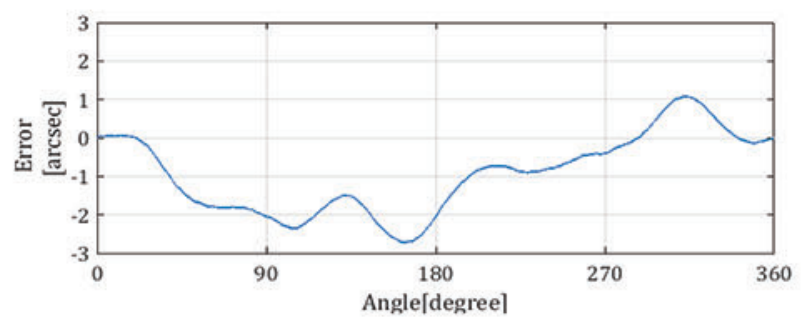

(a) Error curve

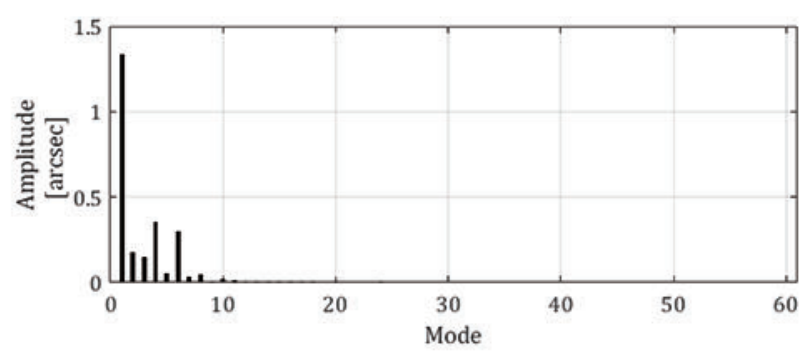

(b) Fourier components in error

Fig. 4 Error by one sensor head on the grating

\subsection{Fundamental Design Concept}

Ideally, the VEMA method can calculate higher-order calibration values with two sensor heads. However, a higher order for the calibration value reduces the angle between the two heads. Consequently, the impact from a mounting error of the sensor heads increases. In addition, the sensor heads have small differences in characteristics, and a higher order increases the 


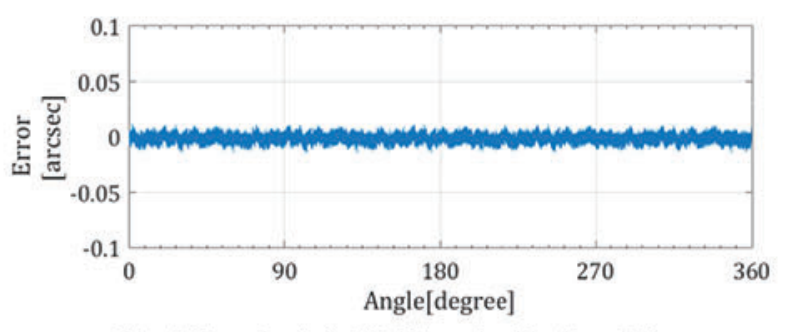

Fig. 5 Error by 2-3-5 SelfA under ideal conditions

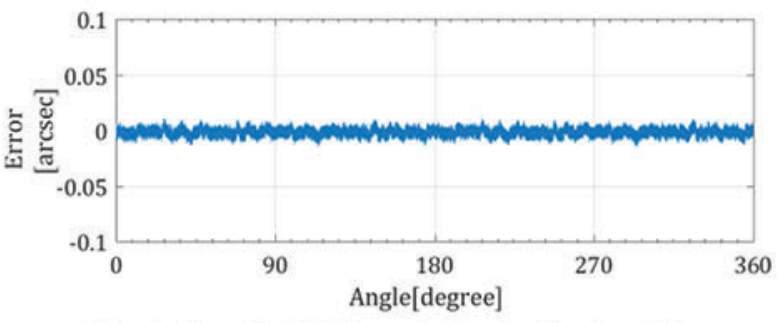

Fig. 6 Error by VEDA method under ideal conditions

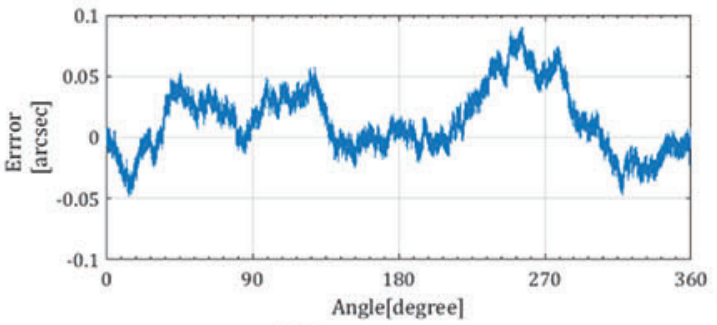

(a) Error curve

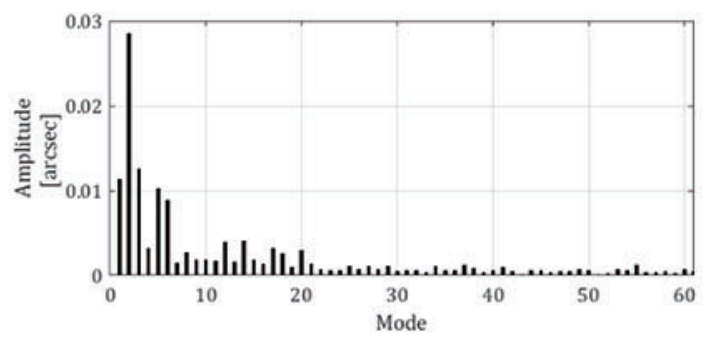

(b) Fourier components in error

Fig. 7 Error by VEDA method with characteristics

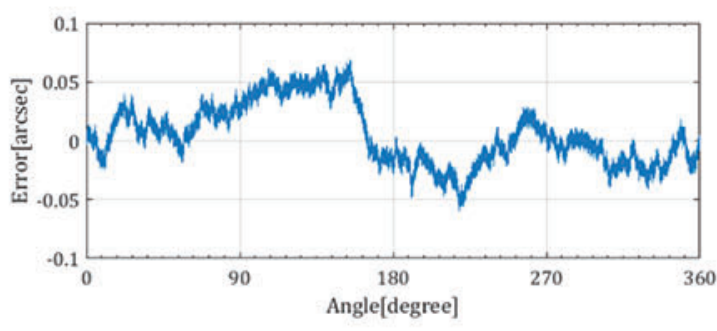

(a) Error curve

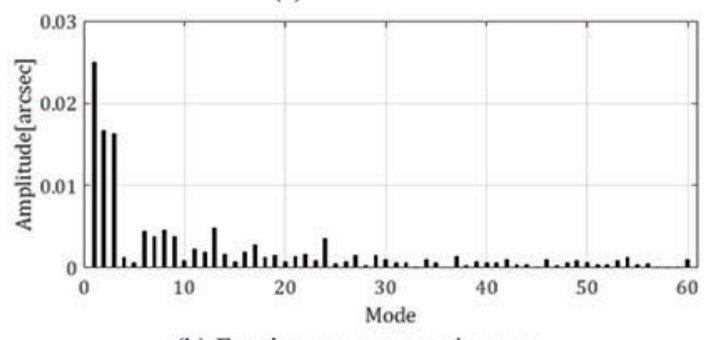

(b) Fourier components in error

Fig. 8 Error by VEDA method with characteristics

effect of the characteristics of each head because more heads are virtually calculated.
Therefore, selecting the appropriate order of calibration is important. More than two sets of heads should be installed, as shown in Fig. 2, and the calibration value should be calculated in the same way as the combined SelfA method ${ }^{7}$. In Fig. 2, a calibration value having an $M=\left(2 \times N_{1} \times N_{2}\right)$ order error can be obtained using four sensors, $H_{0}$ sensor head at 0 rad position, $H_{1}=H^{(2,1)}$ sensor head at $\pi \mathrm{rad}$ position, $H_{2}=$ $H^{\left(N_{1}, 1\right)}$ sensor head at $2 \pi / N_{1}$ rad position and $H_{3}=H^{\left(N_{2}, 1\right)}$ sensor head at $2 \pi / N_{2}$ rad position, where $N_{1}$ and $N_{2}$ is a natural number of 3 or more. Furthermore, the sensor heads should be placed at intervals of $180^{\circ}$ in order to eliminate the effect from axial runout. Fig. 3 shows the arrangement for calibration, where the calibration values of three different orders including the second order are combined. Three pairs of sensor heads were placed at $180^{\circ}$ intervals, for six heads in total. This made it possible to apply the calibration data to all sensor heads. By using the average of all calibrated data as the output of the rotary encoder, the effect from the characteristics of each head was minimized. As a result, the arrangement in Fig. 3 calibrated from a combination of three orders became the arrangement shown in Fig. 3. In this case, the calibration value with the combined method had components other than a multiple of the order at $M=\left(2 \times N_{1} \times N_{2}\right)$.

The detected values over one cycle from all heads needed to be temporarily stored because the phase of the data was shifted during the calibration calculation process. In practice, each head stores $p$ number of values, where $p$ is a multiple of the calibration order. The maximum order for calibration was $p / 2$-order Fourier components for the Nyquist frequency.

\subsection{Feasibility Study by Simulation}

\subsubsection{Conditions of Simulation}

Calibration simulations were run as described in Section 3.1. The SelfA method and multi-reading-head method were chosen for comparison. The calibration conditions are presented below. The multi-reading-head method used six heads (residual error: components of the $32 n$ order ( $n$ : natural number)). The SelfA method had six sensor heads for which the third- and fourth-order components were combined (residual error: $12 n, 3-4$ combined SelfA). The 2-3-5 combined SelfA method had eight heads (residual error: $30 n$ ). The 2-5 combined VEDA method had four heads (residual error: $10 n$ ). The 2-3-5 combined VEDA method had six heads (residual error: $30 n$ ). The 2-5-9 combined VEDA method had six heads (residual error: $90 \mathrm{n}$ ). The calibration simulation was run to determine the accuracy of the grating shown in Fig. 4. Measurements followed the National Standard at AIST ${ }^{8)}$, 9) by using the EDA method and combining the fifth and seventh orders. Therefore, components of the $35 n$-order were missing.

\subsubsection{Effect from Characteristics of Each Head}

The detected value at each head was used to calculate the sum of the error curve based on the scale by which the phase was shifted from the true angular value to the location of each head and the curve of characteristics for each head. Although the characteristics of the error curve for each head varies greatly depending on the principle and manufacturing method of the 


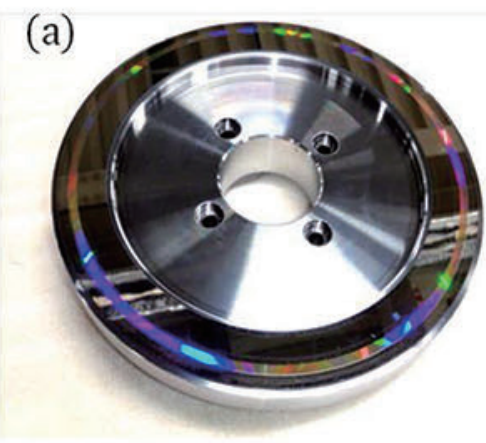

(a) Rotary scale.

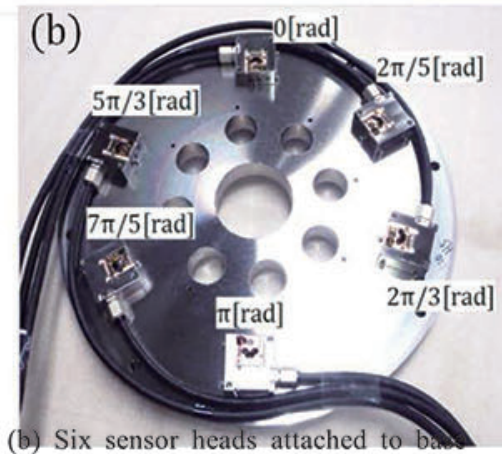

plate (sensor heads arranged according to new method).

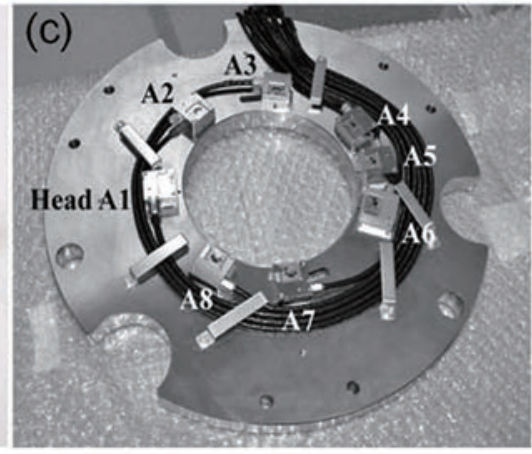

(b) Eight sensor heads attached to base plate (sensor heads arranged according to2-3-5 SelfA method).

Fig. 11 Rotary encoder for prototyping

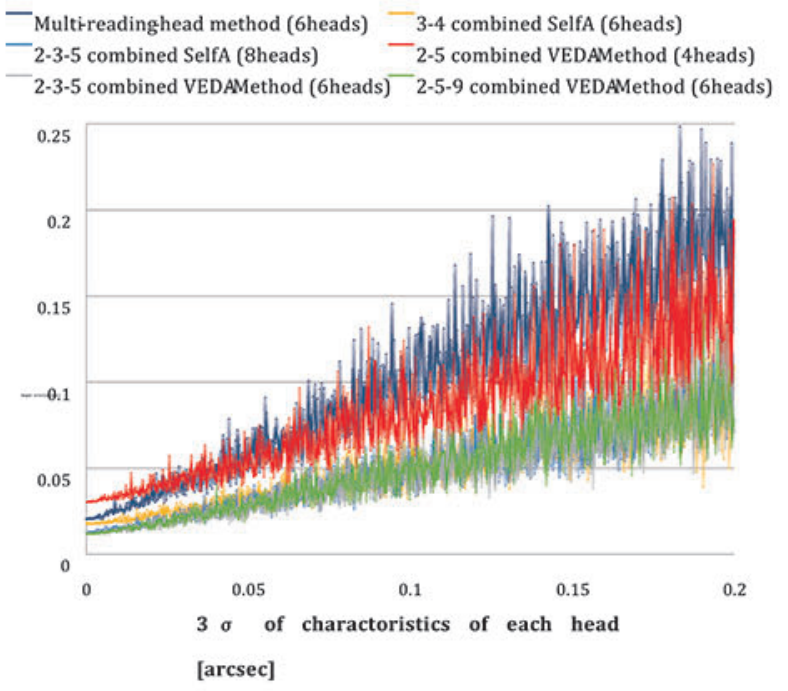

Fig. 9 Amplitude of error calibrated by each method with characteristics

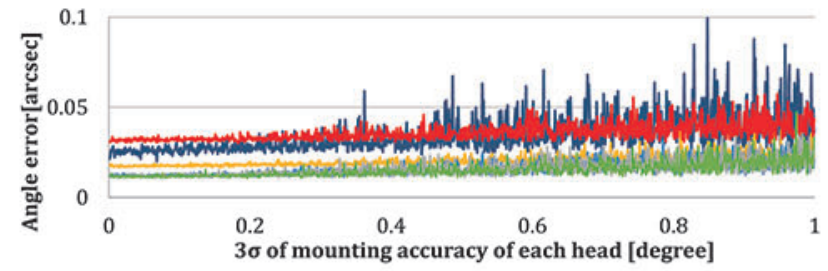

Fig. 10 Amplitude of error after calibration by each method with mounting error

sensor and the scale, in general, the error curve having reproducibility caused by eccentricity, distortion or the like causes a low order to become large, Similarly, even in the characteristics which are the difference of error curve between the sensor heads, the low frequency tends to increase. Brownian noise was used to assume a random signal with a low order like that shown in Fig. 4. Brownian noise is a random signal whose spectral density is proportional to $1 / f^{2}$ with respect to the frequency $f$, that is, its amplitude is proportional to $1 / f$.

Fig. 5 and Fig. 6 shows the results of calibration with the 2-3-5 combined VEDA method and 2-3-5 combined SelfA method without the characteristics or brown noise at $3 \sigma=0.14$. Fig. 7 and Fig. 8 shows the error after calibration with each method as the characteristics changed. The calibration error increased with two pairs of heads (four units). However, it was less than that of the multi-reading-head method. For the 2-3-5 and 2-5-9 VEDA methods, the latter achieved a smaller error without characteristics, but the error increased with the characteristics. Calibration at a higher order increased the effect of the inherent characteristics of each head, as explained in Section 1.2. On the other hand, the 2-3-5 VEDA method with three pairs of heads (six units) achieved an error equivalent to the 2-3-5 combined SelfA method. The VEDA method could compensate for the error with a reasonable level of accuracy and fewer sensor heads.

\subsubsection{Effects of the Mounting Accuracy for Each Head}

Fig. 9 shows the simulation results for each calibration method when each sensor head was assumed to be installed at the proper angle with an accuracy of $3 \sigma$. The mounting error affected calibration by the VEDA method less than the multi-reading-head method and comparable to the combined SelfA method. Fig. 10 indicates that the effects of the mounting error increased with higher-order calibration for the VEDA method. Fewer sensor heads when the calibration order was too high increased the error because of the effects of the characteristics of each head. In contrast, lower-order calibration resulted in inadequate correction of the error from the scale grating.

\subsection{Overall evaluation of feasibility study}

As a result of assuming two kinds of error factors in Section 3.2, in both error factors, the performance is higher than that of SelfA method and Multi-reading-head method when the same number of sensor heads. It was confirmed that a rotary encoder with less influence of characteristics of each sensor and mounting error can be realized.

The number of sensor heads and their locations must be determined by considering the required accuracy, affordable costs, inherent accuracy of the scale, and size of the characteristics of heads. The mounting accuracy was found to be less important than the characteristics of each head.

\section{Verification by Prototyping}

\subsection{Specification of rotary encoder}


The rotary encoder developed for this project has a rotary scale and sensor heads, as shown in Fig. 11. The rotary scale had indent gratings on a glass plate with an outer diameter of $180 \mathrm{~mm}$ and inner diameter of $140 \mathrm{~mm}$. The $1 \mu \mathrm{m}$ pitch contained $2^{19}$ grating lines per cycle. The sensor heads were based on the grating interferometer method and detected gratings to generate $2^{21}$ sinusoidal wave outputs per rotation with a resolution of $2^{37} \times 2^{16}$ divisions through the interpolator. The characteristics of the sensor head were an order of 0.1 arcsec.

To minimize the effect from the characteristics of the head, the VEDA method using three pairs of six heads was chosen from the simulations described in Section 3. The second, third, and fifth orders were selected as the minimum combination of three prime numbers. The Fourier components of the error had a scale of 0.003 or less at an order of 25 th or higher, as shown in Fig. 3. The 30th-order calibration value was enough to meet the target accuracy. To achieve 30th-order calibration with the smallest combination of $N$ possible, the combination of $N=2,3$, and 5 was appropriate. The installation error was sufficiently small when $N$ was 5.

A 2-3-5 combined VEDA method using six sensor heads and a 2-3-5 SelfA method using eight sensors for comparison were prepared. Six of the eight sensor heads for the SelfA method were the same units used for the VEDA method.

\subsection{The experiment}

These heads and rotary scale were installed according to the angular national standard.

Fig. 12 presents the error curve for each of the six heads.

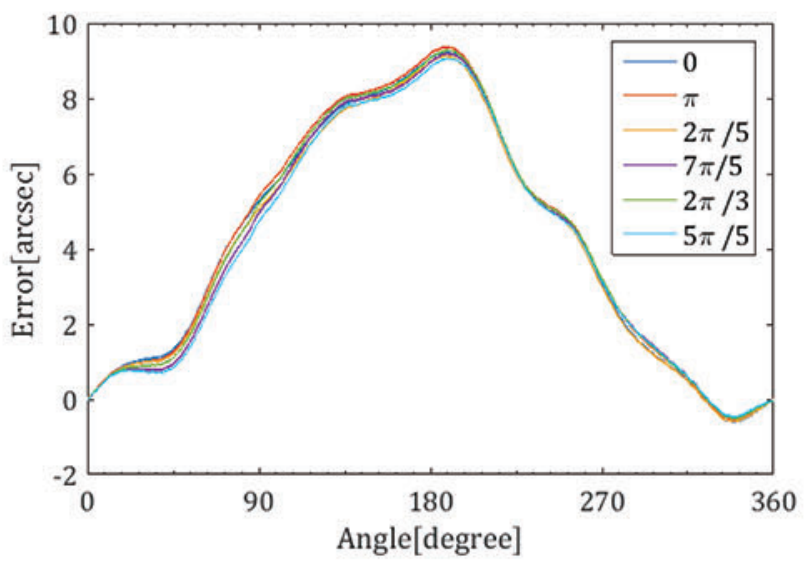

Fig. 12 Phase-shifted error curve of each head

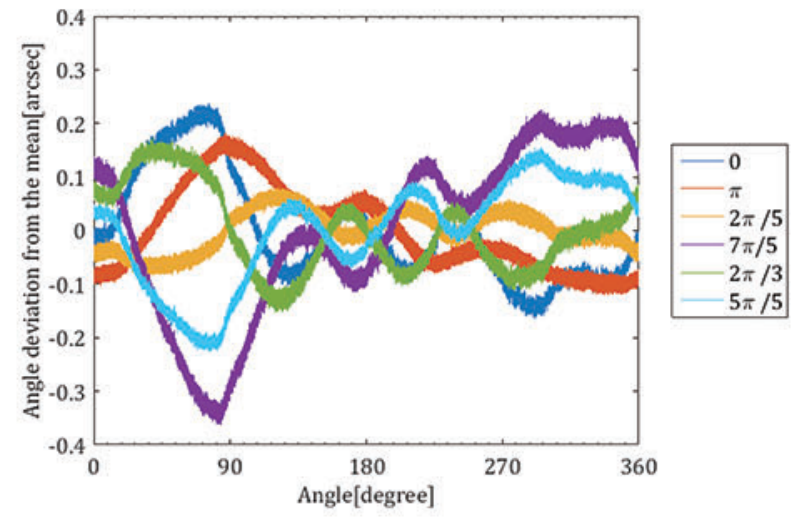

Fig. 13 Characteristics of each head

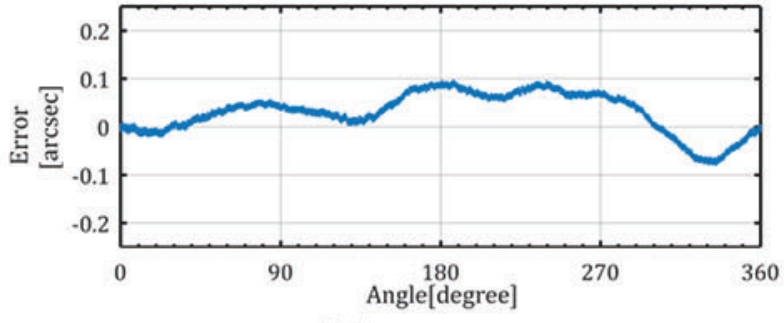

(a) Error curve

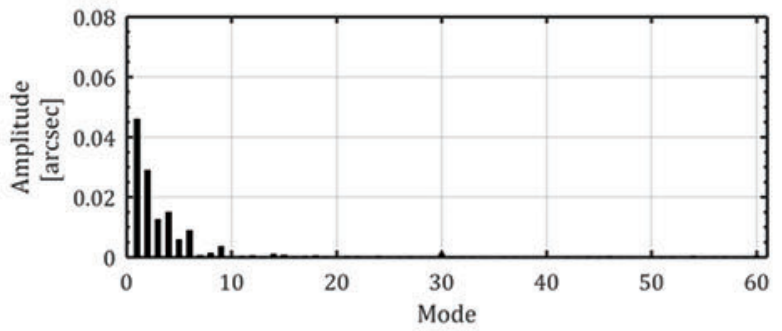

(b) Fourier components in error

Fig. 14 Error calibrated by 2-3-5 combined VEDA method

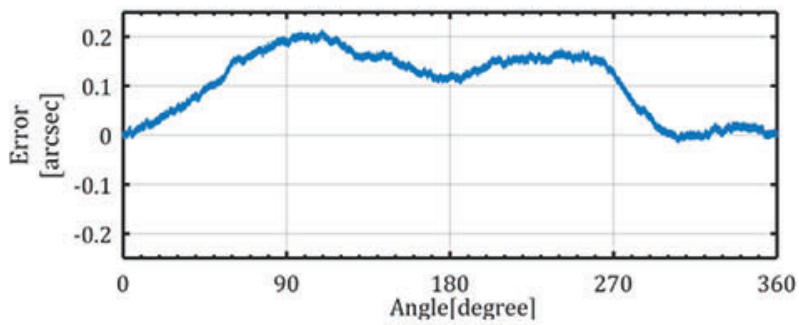

a) Error curve

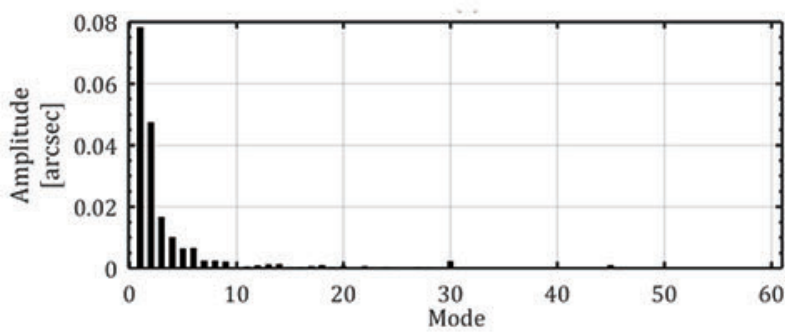

(b) Fourier components in error

Fig. 15 Error calibrated by 2-3-5 combined SelfA

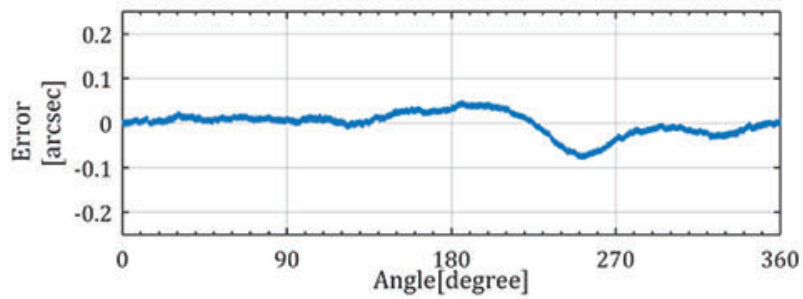

a) Error curve

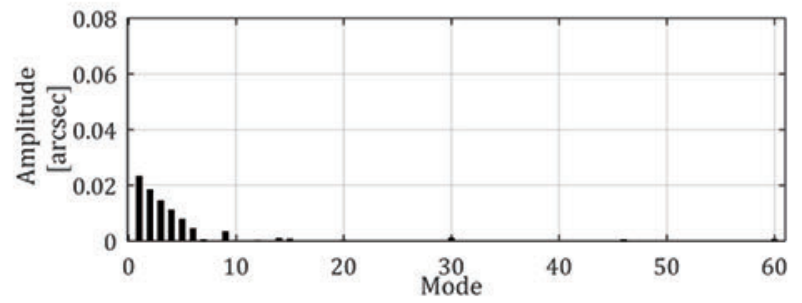

(b) Fourier components in error

Fig. 16 Error calibrated by 2-3-5 combined VEDA method using heads with smaller characteristics 
The phases are shifted to match the angle of the sensor head position. Fig. 13 shows the difference in the error curve after phase adjustment and the average to explain the characteristics of each sensor head as an average deviation of \pm 0.27 arcsec

From the error curve obtained by measuring the sensors one by one, the error when used as an actually calibrated encoder system was calculated. Fig. 14(a) and (b) show the self-calibration results using this error value \pm 0.09 arcsec. Fig. 15(a) and (b) present the results of the combined SelfA method for the eight heads. The average characteristic was \pm 0.31 arcsec with an error of \pm 0.116 arcsec. Thus, the VEDA method achieved higher accuracy. The results confirmed that the VEDA method could compensate as much as the SelfA method with fewer heads. The fewer heads made selecting sensor heads with smaller characteristics easier.

Using the combination that achieves the best accuracy among the 8 error curves, the result is obtained shown in Fig. 16. The average of the characteristics decreased to \pm 0.23 arcsec with an error of \pm 0.067 arcsec.

\section{Conclusion}

A compact, ultra-precise, and high-resolution rotary encoder system was developed by establishing a new self-calibration method that can enhance the measurement accuracy with fewer sensors needed compared to existing method. After the effectiveness of the proposed self-calibration method was verified by simulation, a physical prototype of the rotary encoder system was fabricated for performance evaluation. The prototype rotary encoder based on the grating interferometer method achieved a resolution of $2^{37}$ per cycle with an accuracy of \pm 0.07 arcsec with only six sensor heads.

\section{Acknowledgment}

The authors express their sincere appreciation to Dr Tsukasa Watanabe and National Institute of Advanced Industrial Science and Technology (AIST) for his useful advice and support for the verification by prototyping.

\section{References}

1) G. Goch, W. Knapp, and F. Hartig: Precision Engineering for Wind Energy Systems, Ann. CIRP, 61, 2, (2012) 611.

2) J. C. Aurich, B. Linke, M. Hauschid, M. Carrella, and B. Kirsch: Sustainability of Abrasive Processes, Ann. CIRP, 62, (2012) 653.

3) E. A. Barinova, S. V. Gordeev, E. M. Ivashchenko, and P. A. Pavlov P A: A Method and the Results of Investigating the Random Error of an Optical Angle Encoder, Meas. Tech., 54, (2011) 1075.

4) T. Masuda and M. Kajitani: An Automatic Calibration System for Angular Encoders, Precis. Eng., 11, 2, (1989) 95.

5) T. Watanabe, H. Fujimoto, and T. Masuda: Self-calibratable Rotary Encoder, J. Phys. Conf. Ser., 13, (2005) 240.

6) T. Watanabe, M. Kon, N. Nabeshima, and K. Taniguchi: An Angle Encoder for Super-high Resolution and Super-high Accuracy Using SelfA, Meas. Sci. Technol., 25, 6, (2014) 065002.

7) T. Masuda and M. Kajitani: High Accuracy Calibration System for Angular Encoders, J. Robot. Mechatron., 5, 5 (1993) 448.

8) T. Watanabe, H. Fujimoto, K. Nakayama, T. Masuda, and M. Kajitani: Automatic High-precision Calibration System for Angle Encoder, Proc. SPIE, 4401, (2001) 267.

9) T. Watanabe, H. Fujimoto, K. Nakayama, T. Masuda, and M. Kajitani: Automatic High-precision Calibration System for Angle Encoder (II), Proc. SPIE, 5190, (2003) 400.

10) N. Ishii, K. Taniguchi, K. Yamazaki, and H. Aoyama, Cost-effective and Super-accurate Angular Encoder System with Multi-detecting Heads for Effortless Calibration, ICPE2016, (2016) ,A302-8102 